\title{
RNA Editing Enzymes Modulate the Expression of Hepatic CYP2B6, CYP2C8, and Other Cytochrome P450 Isoforms
}

\author{
Kaori Nozaki, Masataka Nakano, Chika Iwakami, Tatsuki Fukami, and Miki Nakajima \\ Drug Metabolism and Toxicology, Faculty of Pharmaceutical Sciences (K.N., Ma.N., C.I., T.F., Mi.N.), and WPI Nano Life Science \\ Institute (Ma.N., T.F., Mi.N.), Kanazawa University, Kakuma-machi, Kanazawa, Japan
}

Received February 10, 2019; accepted April 8, 2019

\section{ABSTRACT}

A-to-I RNA editing, the most frequent type of RNA editing in mammals, is catalyzed by adenosine deaminase acting on RNA (ADAR) enzymes. Recently, we found that there is a large interindividual variation in the expression of ADAR1 protein in the human livers. In this study, we investigated the possibility that A-to-I RNA editing may modulate the expression of cytochrome P450 (P450), causing interindividual variations in drug metabolism potencies. We found that knockdown of ADAR1 or ADAR2 in HepaRG cells resulted in the decreased expression of CYP2B6 and CYP2C8 mRNA and protein. Knockdown of ADARs significantly decreased the stability of CYP2B6 mRNA but not CYP2C8 mRNA. Luciferase assays revealed that the $3^{\prime}$-untranslated region of CYP2B6 and the promoter region of CYP2C8 would be involved in the decrease in their expression by the knockdown of ADARs. We found that the decreased expression of the hepatocyte nuclear factor $4 \alpha$ (HNF4 $\alpha$ ) protein by the knockdown of ADARs was one of the reasons for the decreased transactivity of CYP2C8. The mRNA levels of other P450 isoforms, such as CYP2A6, 2C9, 2C19, 2D6, and 2E1, which are known to be regulated by HNF4 $\alpha$, were also decreased by ADAR1 or ADAR2 knockdown. Exceptionally, the CYP3A4 mRNA level was significantly increased by ADAR1 knockdown, suggesting the possibility that the change could be due to the change in the expression or function of other regulatory factors. In conclusion, this study revealed that the RNA editing enzymes ADAR1 and ADAR2 are novel regulatory factors of P450-mediated drug metabolism in the human liver.

\section{Introduction}

RNA editing is a post-transcriptional process that changes nucleic acid sequences on RNA. The most frequent type in mammals is A-to-I RNA editing, catalyzed by the adenosine deaminase acting on RNA (ADAR) enzyme (Kim et al., 1994), which converts adenosine in double-stranded RNA to inosine by hydrolytic deamination (Wagner et al., 1989; Nishikura, 2010). Cellular machineries recognize inosine as a guanosine; thus, the conversion possibly changes the resulting amino acid sequence, splicing, microRNA (miRNA) targeting, and maturation (Farajollahi and Maas, 2010). In mammals, there are three members of ADAR: ADAR1, ADAR2, and ADAR3 (Bass et al., 1997). ADAR1 has two isoforms, ADAR1 p150 (a $150 \mathrm{kDa}$ protein) and ADAR1 p110 (a $110 \mathrm{kDa}$ protein). The former is interferon inducible and present in the nucleus and cytoplasm, whereas ADAR1 p110 (110 kDa protein) is constitutively and ubiquitously expressed and present in the nucleus (Patterson and Samuel, 1995; Desterro et al., 2003). ADAR2 is also ubiquitously expressed and localized in the nucleus, whereas the expression of ADAR3 is limited to the brain (Melcher et al., 1996). Although ADAR1 and ADAR2 have A-to-I RNA editing activity,

The work was supported by a Grant-in-Aid for Scientific Research (B) [Grant 18H02573], Grant-in-Aid for Japan Society for the Promotion of Science Fellows [Grant 16J00832], Grant-in-Aid for Early-Career Scientists [Grant 18K14901] from the Japan Society for the Promotion of Science, and World Premier International Research Center Initiative (WPI), Ministry of Education, Culture, Sports, Science and Technology, Tokyo, Japan.

https://doi.org/10.1124/dmd.119.086702.
ADAR3 does not have editing activity (Kim et al., 1994; Chen et al., 2000).

It has been reported that $A D A R 1$ knockout mice are embryonic lethal, caused by severe liver damage (Wang et al., 2000), and $A D A R 2$ knockout mice die within 20 days after birth due to status epilepticus (Higuchi et al., 2000). Thus, RNA editing by ADARs is an essential event for viability. Additionally, the disruption of RNA editing by mutations in the $A D A R$ gene is associated with some diseases (Slotkin and Nishikura, 2013). For example, the abnormal editing of GluR2, an ionotropic glutamate receptor, by the decreased expression or activity of ADAR2 causes amyotrophic lateral sclerosis (Feldmeyer et al., 1999). Mutations in the ADARl gene cause dyschromatosis symmetrica hereditaria, a rare genetic pigmentation disorder (Miyamura et al., 2003). These studies highlight the importance of ADARs in biologic phenomena and homeostasis.

Cytochrome P450s (P450s) are the major drug-metabolizing enzymes, accounting for the biotransformation of $70 \%$ of all drugs in clinical use (Guengerich, 2008). P450s comprise a superfamily, and the CYP1, 2, and 3 families are responsible for drug metabolism in the liver. There are significant interindividual differences in the expression and activity of hepatic P450s, causing variable therapeutic efficacy or adverse events of drugs. Understanding the factors causing interindividual and intraindividual differences in drug metabolism potencies is required for the practice of personalized or precision medicine and the promotion of efficient drug development. The interindividual differences of $\mathrm{P} 450$ s result from intrinsic factors, such as genetic polymorphisms, disease states, sex, and age, as well as extrinsic factors, such as

ABBREVIATIONS: ADAR, adenosine deaminase acting on RNA; AhR, aryl hydrocarbon receptor; HNF4 $\alpha$, hepatocyte nuclear factor $4 \alpha$; LC, liquid chromatography; miRNA, microRNA; P450, cytochrome P450; PCR, polymerase chain reaction; siADAR, small interfering RNA for human adenosine deaminase acting on RNA; siControl, small interfering control; 3'-UTR, 3'-untranslated region. 
exposure to drugs and environmental chemicals, alcohol, smoking, and diet (Zanger and Schwab, 2013). As one of the major mechanisms of the variability in the expression, the transcriptional regulation of $\mathrm{P} 450 \mathrm{~s}$ by transcription factors, including hepatocyte nuclear factors $4 \alpha$ (HNF $4 \alpha$ ), pregnane $\mathrm{X}$ receptor, constitutive androstane receptor, and aryl hydrocarbon receptor (AhR), has greatly been appreciated.

Recently, our research group revealed that there is a large interindividual variation (220-fold) in ADAR1 protein expression in human liver samples (Nakano et al., 2016). This finding prompted us to examine whether the interindividual differences of ADARs may contribute to the hepatic drug metabolism potencies. Recently, we found that ADAR1 negatively regulates AhR expression by creating a binding sequence of miRNA in the $3^{\prime}$-untranslated region ( $3^{\prime}$-UTR). The downregulation of AhR affected CYP1A1, a downstream gene of AhR (Nakano et al., 2016). This was the first study to reveal that A-to-I RNA editing modulates the xenobiotic metabolism. Thereafter, when we searched a database, REDIportal (http://srv00.recas.ba.infn.it/atlas/), we noticed that some $\mathrm{P} 450$ genes and transcriptional factors regulating P450s are registered to be subjected to RNA editing (Nakano and Nakajima, 2018). We expanded our study to investigate whether ADARs regulate the expression and activity of $\mathrm{P} 450$ isoforms in the CYP1, 2, and 3 families. For this purpose, differentiated HepaRG cells were used because they retain P450 expression and activity (Anthérieu et al., 2010) comparable to that in primary human hepatocytes.

\section{Materials and Methods}

Chemicals and Reagents. Phenacetin, acetaminophen, and $\alpha$-amanitin were obtained from Wako Pure Chemicals (Osaka, Japan). Amodiaquine and diclofenac were obtained from Sigma-Aldrich (St. Louis, MO). Hydroxybupropion, bufuralol, and 1'-hydroxybufuralol were obtained from Corning (Corning, NY). The pGL3-basic vector, pNL1.1 vector, and Nano-Glo Dual-Luciferase Reporter Assay System were obtained from Promega (Madison, WI). Lipofectamine RNAiMAX, Silencer Select small interfering RNA for human ADAR [(siADAR), siADAR1 (s1007) and siADAR2 (s1010)], negative control \#1, and Advanced Dulbecco's modified Eagle's medium were obtained from Life Technologies (Carlsbad, CA). Viofectin was purchased from Viogene (Taipei, Taiwan). RNAiso and random hexamers were obtained from Takara (Shiga, Japan). ReverTra Ace was obtained from Toyobo (Osaka, Japan). Primers were commercially synthesized at Integrated DNA Technologies (Coralville, IA). Luna Universal qPCR Master Mix was obtained from New England Biolabs (Ipswich, MA). The mouse anti-human ADAR1, mouse anti-human ADAR2, and mouse anti-human $\beta$-actin monoclonal antibodies were obtained from Santa Cruz Biotechnology (Santa Cruz, CA). The rabbit anti-human CYP2B6 antibody and rabbit anti-human CYP2C8 polyclonal antibody were obtained from Corning. The IRDye 680 goat anti-mouse IgG, goat anti-rabbit IgG, and donkey anti-goat IgG antibodies were obtained from LI-COR Biosciences (Lincoln, NE). The restriction enzymes were obtained from Nippon Gene (Tokyo, Japan) and New England Biolabs. All other chemicals and solvents were of the highest grade commercially available.

Cell Cultures. Human hepatocellular carcinoma-derived HepaRG cells, purchased from KAC (Kyoto, Japan), were cultured in William's E medium supplemented with $10 \%$ FBS (Invitrogen), $100 \mathrm{U} / \mathrm{ml}$ penicillin, $100 \mu \mathrm{g} / \mathrm{ml}$ streptomycin, $5 \mu \mathrm{g} / \mathrm{ml}$ insulin, $2 \mathrm{mM}$ glutamine, and $50 \mu \mathrm{M}$ hydrocortisone hemisuccinate. After 2 weeks, the medium was replaced by the same medium supplemented with $2 \%$ DMSO, and the cell culture was maintained for 2 weeks to differentiate the cells with the maximal functional activities. The medium was exchanged every 2 or 3 days. Human hepatocellular carcinoma-derived Huh-7 cells, obtained from Riken Gene Bank (Tsukuba, Japan), were cultured in Dulbecco's modified Eagle's medium (Nissui Pharmaceutical, Tokyo, Japan) containing $10 \% \mathrm{FBS}$. These cells were cultured at $37^{\circ} \mathrm{C}$ under the atmospheric conditions of $5 \% \mathrm{CO}_{2}$ and $95 \%$ air.

Transfection of Small Interfering RNA for ADAR1 or ADAR2 into HepaRG of Huh-7 Cells and the Preparation of Cell Homogenate, Total RNA, and Nuclear Extracts. The differentiated HepaRG cells were seeded into six-well plates and transfected with $50 \mathrm{nM}$ siADAR1 or siADAR2 using Lipofectamine RNAiMAX. After incubation for 72 hours in DMSO-free medium, the cells were harvested. One-half of the cells were suspended in Tris-GlycineEDTA buffer [10 mM Tris-HCl, $20 \%$ glycerol, and $1 \mathrm{mM}$ EDTA (pH 7.4)], disrupted by freeze/thawing three times, and homogenized. The other half of the cells was used to prepare total RNA using RNAiso. Huh-7 cells were seeded into six-well plates and transfected with $5 \mathrm{nM}$ siADAR1 or siADAR2 using Lipofectamine RNAiMAX. After incubation for 72 hours, the cells were harvested and used to prepare nuclear extracts following the research by Dignam et al. (1983) with slight modification. Briefly, Huh-7 cells were suspended with five volumes of buffer A (10 mM HEPES, pH 7.6, $10 \mathrm{mM}$ $\mathrm{KCl}, 1.5 \mathrm{mM} \mathrm{MgCl}_{2}, 0.5 \mathrm{mM}$ dithiothreitol, and $0.05 \% \mathrm{NP} 40$ ), and centrifuged at $3000 \mathrm{rpm}$ at $4{ }^{\circ} \mathrm{C}$ for 5 minutes. The supernatant was removed and two volumes of buffer A were added to the pellet. The suspension was centrifuged at $3000 \mathrm{rpm}$ at $4^{\circ} \mathrm{C}$ for 5 minutes. The supernatant was removed and two volumes of buffer $\mathrm{C}$ (20 mM HEPES, pH 7.8, $420 \mathrm{mM} \mathrm{KCl}, 0.2 \mathrm{mM}$ EDTA, $1.5 \mathrm{mM} \mathrm{MgCl}_{2}, 1 \mathrm{mM}$ dithiothreitol, and $10 \%$ glycerol) were added to the pellet. After incubation for 30 minutes at $4{ }^{\circ} \mathrm{C}$, the suspension was centrifuged at $15,000 \mathrm{rpm}$ at $4^{\circ} \mathrm{C}$ for 30 minutes and the supernatant was recovered as nuclear extracts.

SDS-PAGE and Western Blot Analysis. The cell homogenates (20 $\mu \mathrm{g}$ for ADAR1, CYP2B6, $10 \mu \mathrm{g}$ for $\beta$-actin, and $30 \mu \mathrm{g}$ for CYP2C8) and nuclear extracts $(20 \mu \mathrm{g}$ for $\mathrm{HNF} 4 \alpha)$ were separated by $7.5 \%$ SDS-PAGE and transferred to an Immobilon-P transfer membrane (Millipore, Billerica, MA). For ADAR2 analysis, $50 \mu \mathrm{g}$ of the cell homogenate was separated by $7.5 \%$ SDS-PAGE and transferred to a Protran nitrocellulose membrane (Whatman Gmbh, Dassel, Germany). The membranes were probed with the primary antibody and then with the fluorescent, dye-conjugated secondary antibodies. The bands were detected using the Odyssey Infrared Imaging system (LI-COR Biosciences). The ADAR1, ADAR2, CYP2B6, and CYP2C8 protein levels were normalized to the $\beta$-actin protein levels.

Real-Time Reverse Transcription Polymerase Chain Reaction. The cDNA was synthesized from total RNA using ReverTra Ace. A $1 \mu$ l portion of the reverse-transcribed mixture was added to the polymerase chain reaction (PCR) mixture containing 5 pmol of each primer and $10 \mu 1$ of the Luna Universal qPCR Mix in a final volume of $20 \mu$ l. The sequences of the primers and PCR conditions are shown in Table 1. Real-time reverse transcription PCR was performed using Mx3000P (Stratagene). Each P450 mRNA level was normalized to the $\beta$-actin mRNA level.

Measurement of P450 Activity. The differentiated HepaRG cells were transfected with siADARs as described previously. After 72 hours, the medium was replaced with FBS-free Advanced Dulbecco's modified Eagle's medium containing $100 \mu \mathrm{M}$ phenacetin (CYP1A2), $2 \mu \mathrm{M}$ amodiaquine (CYP2C8), $50 \mu \mathrm{M}$ dicrofenac (CYP2C9), and $20 \mu \mathrm{M}$ bufuralol (CYP2D6). After incubations at $37^{\circ} \mathrm{C}$ for 4 hours (CYP1A2), 30 minutes (CYP2C8), 3 hours (CYP2C9), and 1 hour (CYP2D6), the medium was collected and an equal volume of acetonitrile was added to the medium. After removing the protein by centrifugation at $15,000 \mathrm{rpm}$ for 5 minutes, a portion of supernatant was subjected to liquid chromatography (LC)-tandem mass spectrometry. The LC equipment (Shimadzu, Kyoto, Japan) contained a CBM-20A controller, LC-20AD pumps, a SIL-20AC HT autosampler, a CTO-20AC column oven, and an SPD-20A UV detector equipped with a Develosil ODS-UG-3 column $(3 \mu \mathrm{m}, 4.6 \times 150 \mathrm{~mm}$; Nomura Chemical, Seto, Japan). The column temperature was set at $40^{\circ} \mathrm{C}$, and the flow rate was $0.2 \mathrm{ml} / \mathrm{min}$. The mobile phase was water containing $0.1 \%$ formic acid (A) and acetonitrile containing $0.1 \%$ formic acid (B). The conditions for elution were as follows: $20 \%-80 \%$ B (0-2 minutes), $80 \%$ B (2-5 minutes), and $20 \%$ B (5-7 minutes). The LC was connected to an LC-MS 8040 (Shimadzu). The metabolites were monitored in the multiple reactions monitoring mode. The mass spectrometry conditions are shown in Table 2 . The analytical data were processed using LabSolutions (version $5.82 \mathrm{SP1}$; Shimadzu).

Evaluation of the Stability of P450 mRNA. HepaRG cells were transfected with siADARs as described previously. After 24 hours, the cells were treated with $10 \mu \mathrm{g} / \mathrm{ml}$ of $\alpha$-amanitin, an inhibitor of RNA polymerase II. The total RNA was prepared 0,1,2, 4, and 6 hours later. The CYP2B6 and CYP2C8 mRNA levels were determined by real-time reverse transcription PCR, as described previously.

Reporter Plasmid Construction. The $3^{\prime}$-UTR of CYP2B6, from +1461 to 3052, was amplified by PCR using genomic DNA as a template. The nucleotide numbers refer to the transcription start site as +1 . The following primer set was used: 5'-CGT GGC CCC AGA AGA CAT CGA TC- 3' and 5'-GGG AGG TCA 
TABLE 1

Sequence of primers used for reverse transcription PCR

\begin{tabular}{|c|c|c|c|c|c|}
\hline Target & & Sequence & Annealing Temperature & Extension Time & Step \\
\hline & & & ${ }^{\circ} \mathrm{C}$ & $s$ & \\
\hline \multirow[t]{2}{*}{$\mathrm{CYP} 1 \mathrm{~A} 2^{a}$} & $\mathrm{~S}$ & 5'- CCA ACG TCA TTG GTG CCA TG $-3^{\prime}$ & 57 & 20 & 3 \\
\hline & AS & $5^{\prime}-$ GTG ATG TCC CGG ACA CTG TTC $-3^{\prime}$ & & & \\
\hline \multirow{2}{*}{ CYP2A $6^{b}$} & $\mathrm{~S}$ & $5^{\prime}-$ AAA GAG TTC CTG TCA CTG TTG C $-3^{\prime}$ & 64 & 20 & 2 \\
\hline & AS & 5'- GCT GAC GGT CTC GGT GCC CC -3' & & & \\
\hline \multirow[t]{2}{*}{ CYP2B6 } & $\mathrm{S}$ & 5'- CTT GCG GGG ATA TGG TGT GA -3' & 57 & 20 & 3 \\
\hline & AS & $5^{\prime}-$ TCA AAC AGC TGG CCG AAT AC $-3^{\prime}$ & & & \\
\hline \multirow{2}{*}{$\mathrm{CYP} 2 \mathrm{C} 8^{c}$} & $\mathrm{~S}$ & $5^{\prime}-$ AGA TCA GAA TTT TCT CAC CC $-3^{\prime}$ & 60 & 20 & 2 \\
\hline & AS & $5^{\prime}-$ AAC TTC GTG TAA GAG CAA CA - $3^{\prime}$ & & & \\
\hline \multirow[t]{2}{*}{$\mathrm{CYP} 2 \mathrm{C} 9^{c}$} & $\mathrm{~S}$ & 5'- CAG ATC TGC AAT AAT TTT TCT C -3' & 50 & 15 & 3 \\
\hline & AS & $5^{\prime}$ - CTT TCA ATA GTA AAT TCA GAT G - $3^{\prime}$ & & & \\
\hline \multirow[t]{2}{*}{$\mathrm{CYP} 2 \mathrm{C} 19^{c}$} & $\mathrm{~S}$ & $5^{\prime}-$ ATT GAA TGA AAA CAT CAG GAT TG $-3^{\prime}$ & 64 & 20 & 2 \\
\hline & AS & 5'- GAG GGT TGT TGA TGT CCA TC -3' & & & \\
\hline \multirow[t]{2}{*}{ CYP2D6 ${ }^{c}$} & $\mathrm{~S}$ & $5^{\prime}-$ GGT GTG ACC CAT ATG ACA TC $-3^{\prime}$ & 60 & 15 & 3 \\
\hline & AS & $5^{\prime}-$ CTC CCC GAG GCA TGC ACG - $3^{\prime}$ & & & \\
\hline \multirow[t]{2}{*}{$\mathrm{CYP}_{2} \mathrm{E}^{d}$} & $\mathrm{~S}$ & $5^{\prime}-$ ACG GTA TCA CCG TGA CTG TGG $-3^{\prime}$ & 58 & 20 & 2 \\
\hline & AS & $5^{\prime}-$ GCA TCT CTT GCC TAT CCT TGA $-3^{\prime}$ & & & \\
\hline \multirow[t]{2}{*}{ CYP3A4 } & $\mathrm{S}$ & 5'- GTA TGG AAA AGT GTG GGG CT -3' & 60 & 20 & 2 \\
\hline & AS & 5'- GAC CAT CTC CTT GAG TTT TCC A - $3^{\prime}$ & & & \\
\hline \multirow{2}{*}{ CYP3A5 ${ }^{c}$} & $\mathrm{~S}$ & $5^{\prime}$ - AGT GTT CTT TCC TTC ACT TTA -3' & 60 & 20 & 2 \\
\hline & AS & 5'- TTC AAC ATC TTT CTT GCA AGT -3' & & & \\
\hline \multirow[t]{2}{*}{$\beta$-Actin ${ }^{e}$} & $\mathrm{~S}$ & $5^{\prime}-$ TCA CCC TGA AGT ACC CCA TC $-3^{\prime}$ & 64 & 30 & 2 \\
\hline & AS & 5'- GAT AGC ACA GCC TGG ATA GC -3' & & & \\
\hline
\end{tabular}

S, Sense primer; AS, Antisense primer.

${ }^{a}$ Iwanari et al. (2002).

${ }^{b}$ Nakajima et al. (2006).

${ }^{c}$ Katoh et al. (2004).

${ }^{d}$ Mohri et al. (2010)

${ }^{e}$ Oda et al. (2012).

GGC TTT AGA G-3' ${ }^{\prime}$. The fragment was inserted into the pGL3p vector at the XbaI site downstream from the luciferase gene. The constructed luciferase reporter plasmid was termed pGL3p/2B6 3'-UTR.

The $5^{\prime}$-flanking region of the $C Y P 2 C 8$ gene, from -2491 to -1 , was amplified by PCR using genomic DNA as a template. The following primer set was used: $5^{\prime}-$ CGC GGT ACC TCA CAA CCC TAT CCA AGC TG- $3^{\prime}$ and $5^{\prime}$-TTG AAG CTT AAA GTA AAC AAT CAC CTA GAT CCA AT- $3^{\prime}$. The fragment was inserted into the pGL3b vector via the KpnI and Hind III restriction sites to construct the pGL3b/2C8-2491 plasmid. This plasmid was digested by SphI and Hind III to obtain a fragment containing the sequence from -514 to -1 , and the fragment was cloned into the pGL3b vector to construct the pGL3b/2C8-514 plasmid. In the HNF $4 \alpha$ binding site from -57 to -45 base pairs, the mutations were introduced from -53 to -48 base pairs by inverse PCR, followed by an In-Fusion reaction using the In-Fusion HD Enzyme Premix (Takara). For the inverse PCR, the following PCR primer set was used: $5^{\prime}$-TGG GCG TTT CAC TCA GAA AAA AAG TAT AAA - $3^{\prime}$ and $5^{\prime}$-GTG GTG AAA CGC CCA TGG ATA GAA ATA AAA TGT $-3^{\prime}$. The underlined nucleotides represent the mutated sequence. The obtained plasmid was termed the pGL3b/2C8 MT plasmid.

Luciferase Assay. The various pGL3 luciferase reporter plasmids were transfected with the pNL 1.1 plasmid into Huh-7 cells. Briefly, the cells were seeded into 96-well plates. After 24 hours, $5 \mathrm{nM}$ siADAR1 or siADAR2 was transfected using Lipofectamine RNAiMAX. After 24 hours, $100 \mathrm{ng}$ of the pGL3b plasmid and $100 \mathrm{pg}$ of the pNL 1.1 plasmid were transfected using Viofectin. After 48 hours, the luciferase activity was measured with a luminometer (Wallac, Turku, Finland) using the Nano-Glo Dual-Luciferase Reporter Assay System.

Assessment of RNA Editing Level in the CYP2B6 3'-UTR. To analyze RNA editing in the $3^{\prime}$-UTR of CYP2B6, direct sequence analysis of the PCR product was performed following our previous study (Nakano et al., 2016). The 3'-UTR of CYP2B6 was amplified by PCR using cDNA derived from HepaRG cells as a template. The following primer sets were used to amplify the $3^{\prime}$-UTR of CYP2B6: the forward primer 5'-CGT GGC CCC AGA AGA CAT CGA TC-3' and reverse primer 5'-TGA CAT CAG GAG TTC AAG ACC-3', or the forward primer $5^{\prime}$-GGT GAT TCT CCT AGC TCC AA- $3^{\prime}$ and reverse primer $5^{\prime}$-CAT GAA AGC AAC CAG ACA CAA- $3^{\prime}$. Specific products were purified and subjected to direct sequencing.
Statistical Analyses. Statistical significance was determined by ANOVA followed by Tukey's method. A value of $P<0.05$ was considered statistically significant.

\section{Results}

CYP2B6 Expression Level Was Decreased by the Knockdown of ADARs. To examine the possibility that hepatic $\mathrm{P} 450$ s are regulated by ADARs, either siADAR1 or siADAR2 was transfected into HepaRG cells. The siADAR1 construct used in this study can target both ADAR1 p110 and ADAR1 p150. As shown in Fig. 1A, ADAR1 p110 was significantly decreased [ $91 \%$ of small interfering control (siControl)] by the transfection of siADAR1 but not siADAR2. The ADAR1 p150 protein was not detected. The ADAR2 protein was significantly decreased ( $77 \%$ and $56 \%$ of siControl, respectively) by the transfection of siADAR2 and siADAR1 (Fig. 1B). Thus, we confirmed that either the ADAR1 or ADAR2 protein was successfully knocked down.

Next, we evaluated the expression levels of the P450 isoforms in the ADAR knockdown cells. We found that the CYP2B6 mRNA (73\% and $68 \%$ of siControl, respectively) and protein $(65 \%$ and $53 \%$ of siControl, respectively) (Fig. 2) levels were significantly decreased by

TABLE 2

Conditions of liquid chromatography-tandem mass spectrometry

\begin{tabular}{|c|c|c|c|c|}
\hline \multirow{2}{*}{ Isoform } & \multirow{2}{*}{ Metabolite } & \multicolumn{2}{|c|}{$m / z$} & \multirow{2}{*}{ Collision Energy } \\
\hline & & Precursor Ion & Production & \\
\hline & & & & $e V$ \\
\hline CYP1A2 & Acetaminophen & 152.05 & 110.00 & -16 \\
\hline CYP2C8 & $N$-Desethylamodiaquine & 328.10 & 283.05 & -19 \\
\hline CYP2C9 & $4^{\prime}$-Hydroxydiclofenac & 311.15 & 267.10 & 10 \\
\hline CYP2D6 & 1'-Hydroxybufuralol & 278.35 & 186.05 & -18 \\
\hline
\end{tabular}

$\mathrm{m} / \mathrm{z}$, mass-to-charge ratio. 
A

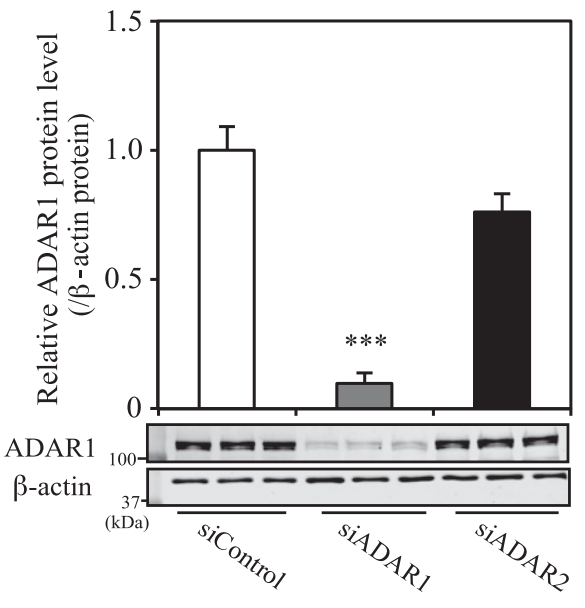

B

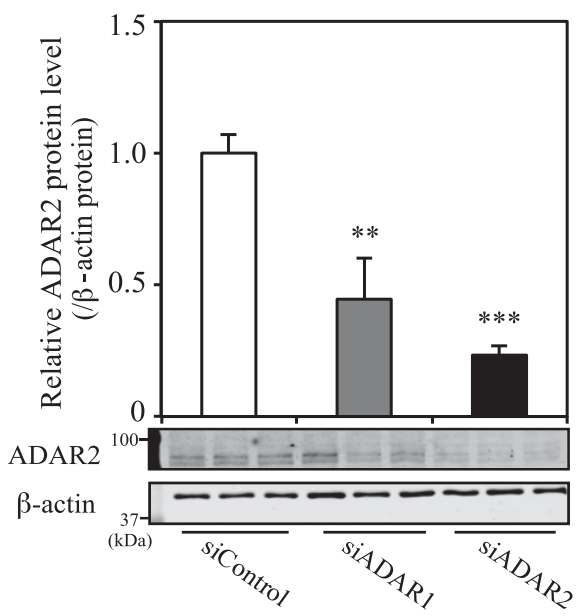

Fig. 1. Knockdown of ADAR1 or ADAR2 in differentiated HepaRG cells. ADAR1 (A) and ADAR2 (B) protein levels in siADAR1- or siADAR2-transfected HepaRG cells were determined by western blotting and normalized to the $\beta$-actin levels. The values represent the levels relative to the siControl. Each column represents the mean \pm S.D. of three independent experiments. ${ }^{* *} P<0.01 ; * * * P<0.001$, compared with siControl. the knockdown of ADAR1 or ADAR2. These results suggest that ADARs positively regulate the expression level of CYP2B6.

ADARs Stabilize CYP2B6 mRNA by Targeting the $\mathbf{3}^{\prime}$-UTR. We investigated whether the decrease in CYP2B6 expression by ADAR knockdown was due to enhanced mRNA degradation. In the presence of $\alpha$-amanitin, a transcriptional inhibitor, the CYP2B6 mRNA level remained unchanged until after 6 hours in the siControl-transfected cells, indicating that the CYP2B6 mRNA is stable. By the knockdown of ADAR1 or ADAR2, the CYP2B6 mRNA levels were significantly decreased (30\% and $40 \%$ of siControl, respectively) 6 hours after treatment with $\alpha$-amanitin (Fig. 3A), indicating that ADARs positively regulate the CYP2B6 mRNA expression by stabilizing the mRNA. Next, to examine the involvement of the $3^{\prime}$-UTR in the decrease in CYP2B6 expression by the knockdown of ADARs, a luciferase assay using a reporter plasmid containing the $3^{\prime}$-UTR was performed (Fig. 3B). Because the reporter plasmid could not efficiently be transfected into the differentiated HepaRG cells, we used Huh-7 cells instead of the HepaRG cells. The luciferase activities of pGL3p/2B6 3'-UTR were lower than those of pGL3p/empty. It is common that the luciferase activity of the reporter plasmid containing the $3^{\prime}$-UTR is lower than that of the empty plasmid because $3^{\prime}$-UTR includes miRNA binding sites. Actually, it has been reported that miR-25-3p suppresses the expression of CYP2B6 in HepaRG cells via binding to the 3'-UTR (Jin et al., 2016). When the reporter plasmid was transfected with small interfering RNA into Huh-7 cells, the luciferase activity of pGL3p/2B6 3'-UTR was decreased (30\% of siControl) by the knockdown of ADAR2 but not of ADAR1, suggesting that ADAR2 apparently regulates the CYP2B6 expression by targeting the $3^{\prime}$-UTR.

No A-to-I RNA Edited Sites Were Found in the $3^{\prime}$-UTR of CYP2B6. In REDIportal, RNA editing sites are registered within the two Alu elements in the $3^{\prime}$-UTR of CYP2B6. To examine whether the 3'-UTR of CYP2B6 is subjected to RNA editing, we performed direct sequencing. In the results, no edited sites were detected in the two Alu elements located in the $3^{\prime}$-UTR of CYP2B6 (data not shown). Therefore, the change in the CYP2B6 expression by the knockdown of ADAR1 or ADAR2 appears to be not relevant with the RNA editing of CYP2B6 by itself.

CYP2C8 Expression Level and Activity Were Decreased by the Knockdown of ADARs. We investigated whether the knockdown of ADARs affects other P450 expression levels, focusing on CYP2C8 as one example. As shown in Fig. 4, A and B, the CYP2C8 mRNA (66\% and $54 \%$ of siControl, respectively) and protein (77\% and $64 \%$ of siControl, respectively) levels were significantly decreased by the knockdown of ADAR1 or ADAR2. To examine if the CYP2C8 activity
A

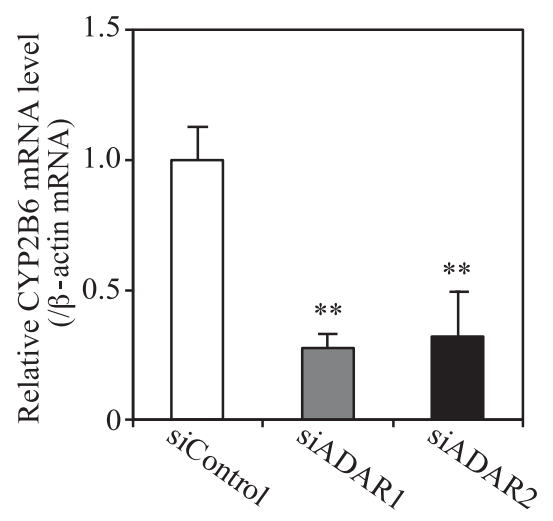

B

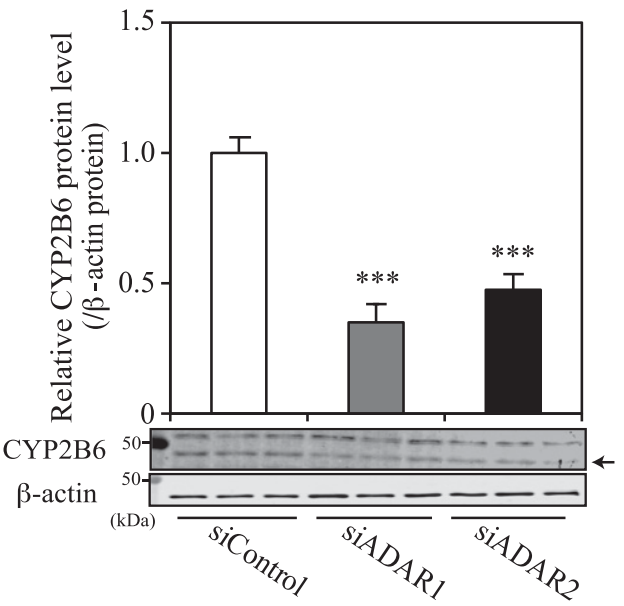

Fig. 2. Effects of ADAR1 or ADAR2 knockdown on the expression of CYP2B6 mRNA and protein in differentiated HepaRG cells. CYP2B6 mRNA (A) and protein (B) levels in siADAR1- or siADAR2transfected HepaRG cells were determined by real-time reverse transcription PCR and western blot analysis, respectively, and normalized to the $\beta$-actin levels. It was confirmed that the lower band represents CYP2B6 by comparison with the band observed with a commercially available recombinant CYP2B6 (data not shown). The values represent the levels relative to the siControl. Each column represents the mean \pm S.D. of three independent experiments. $* * P<0.01$; *** $P<$ 0.001 , compared with siControl. 
A

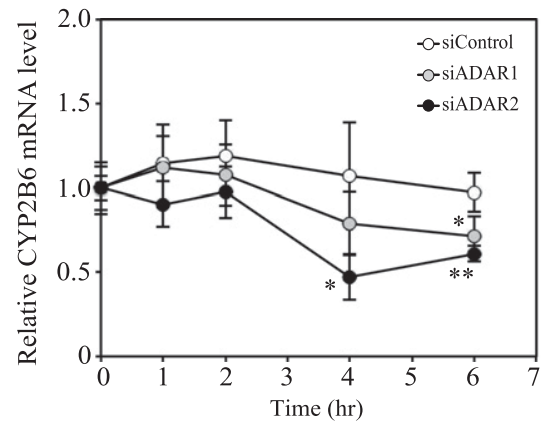

B

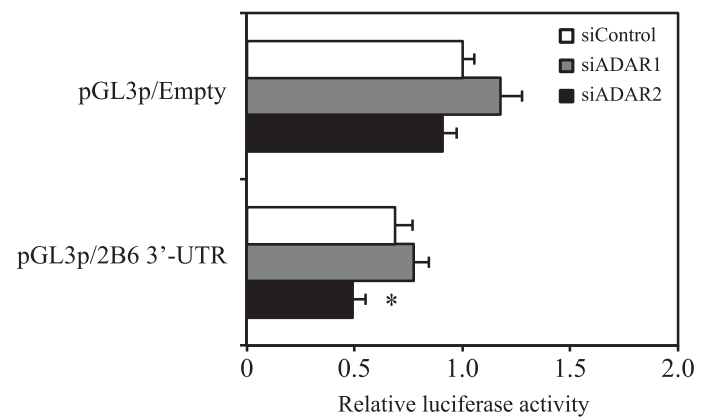

Fig. 3. Effects of ADAR1 or ADAR2 knockdown on the stability of CYP2B6 mRNA and the use of luciferase assays to examine the significance of the 3 '-UTR. (A) siADAR1- or siADAR2-transfected HepaRG cells were treated with $10 \mu \mathrm{g} / \mathrm{ml}$ of $\alpha$-amanitin and the CYP2B6 mRNA level was determined by real-time reverse transcription PCR. The values are expressed as relative to the values of the CYP2B6 mRNA level at 0 hours. Each point represents the mean \pm S.D. of three independent experiments. $* P<0.05$; $* * P<0.01$. (B) Huh-7 cells were transfected with $5 \mathrm{nM}$ siADAR1 or siADAR2. After 24 hours, the reporter plasmids were transfected. The values are expressed as the activity relative to that of the pGL3p plasmid/siControl. Each column represents the mean \pm S.D. of three independent experiments. ${ }^{*} P<0.05$, compared with siControl. is also decreased, the amodiaquine $N$-desethylase activity was evaluated by LC-tandem mass spectrometry. The activity (64\% and $40 \%$ of siControl, respectively) was significantly decreased by the knockdown of ADAR1 and ADAR2, although the latter case did not reach a statistically significant difference (Fig. 4C). These results suggest that ADARs positively regulate the CYP2C8 expression and activity.

ADARs Positively Regulate the Transactivity of CYP2C8 via HNF4 $\boldsymbol{\alpha}$. In the same way as CYP2B6, we investigated whether the knockdown of ADARs results in a decrease in the CYP2C8 mRNA stability. Unlike the stability of CYP2B6, the stability of CYP2C8 mRNA was not changed by ADAR1 or ADAR2 knockdown (Fig. 5A). Then, to investigate whether the knockdown of ADARs affects the transactivity of CYP2C8, a luciferase assay using reporter plasmids containing the promoter region of CYP2C8 was performed. When the pGL3b/2C8-2491 plasmid was used, the luciferase activity was decreased (32\% of siControl) by ADAR2 knockdown but not by ADAR1 knockdown. In contrast, the luciferase activity of the pGL3b/2C8-514 plasmid (30\% and $49 \%$ of siControl, respectively) was decreased by ADAR1 or ADAR2 knockdown (Fig. 5B). We noticed that there is a binding site of $\mathrm{HNF} 4 \alpha$ in the upstream region of -57 to -45 (Ferguson et al., 2005) in the CYP2C8 gene. To examine whether the disruption of
HNF $4 \alpha$ binding may counteract the siADAR-dependent decrease in the transactivity, a luciferase assay using the plasmid containing a mutation in the response element of $\mathrm{HNF} 4 \alpha$ was performed. When pGL3b/2C8-514MT was transfected, the luciferase activity was not changed by the knockdown of ADAR1 or ADAR2 (Fig. 5B).

Next, we examined whether the decrease in CYP2C8 transactivity was due to the decreased expression of HNF $4 \alpha$. As shown in Fig. 5, C and $\mathrm{D}$, we found that the $\mathrm{HNF} 4 \alpha$ protein $(63 \%$ and $79 \%$ of siControl, respectively) level was significantly decreased by ADAR1 or ADAR2 knockdown, although the HNF $4 \alpha$ mRNA level was not decreased. These results suggest that the decrease in CYP2C8 expression by the knockdown of ADAR1 or ADAR2 could be attributed to the repression of $\mathrm{HNF} 4 \alpha$ expression.

Effects of ADAR Knockdown on the Other P450 mRNA Expression Levels and Activities in Differentiated HepaRG Cells. It is known that $\mathrm{HNF} 4 \alpha$ transactivates not only CYP2B6 and $2 \mathrm{C} 8$ but also CYP2A6, 2C9, 2C19, 2D6, 2E1, and 3A4 (Kamiyama et al., 2007; Chen et al., 2018). Given that the $\mathrm{HNF} 4 \alpha$ protein expression is regulated by ADARs, we investigated whether the expression of these P450 mRNAs is also modulated in siADAR1- or siADAR2-transfected HepaRG cells. As shown in Fig. 6, A-F and H, the CYP1A2 (20\%
A

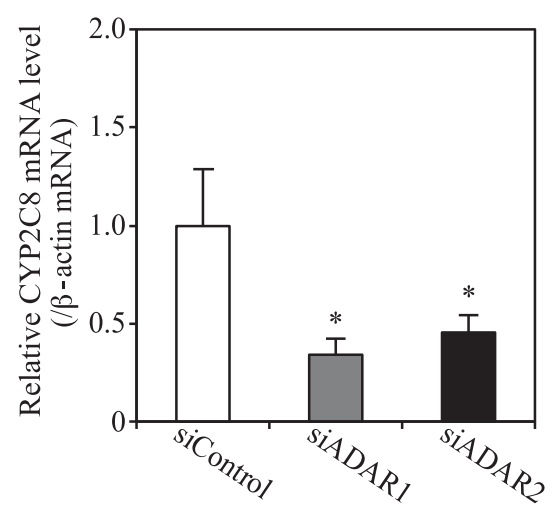

B

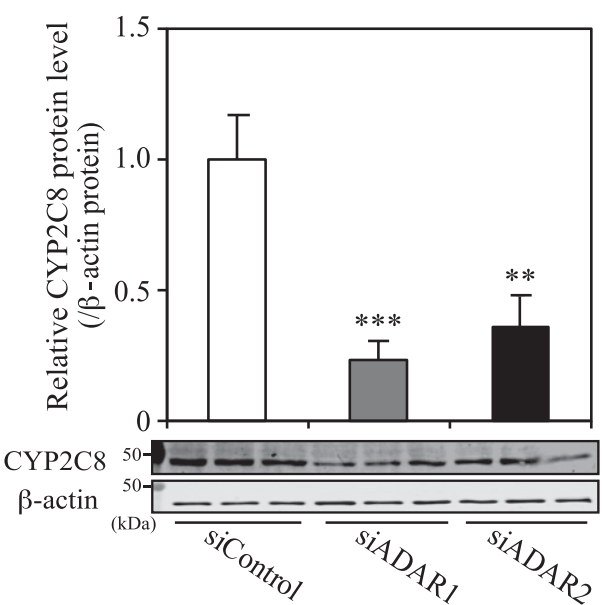

C

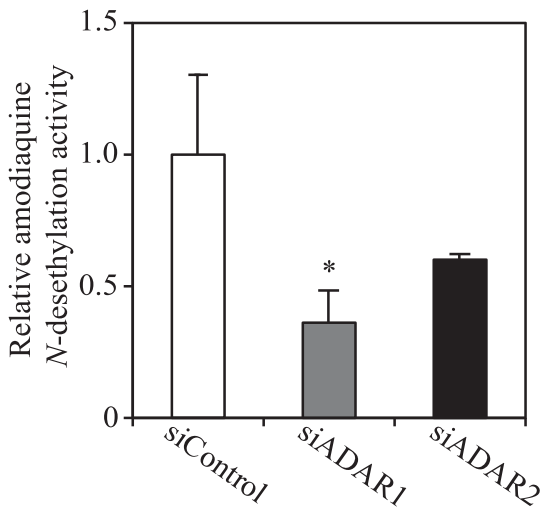

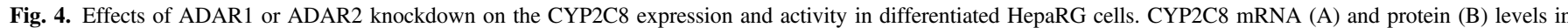

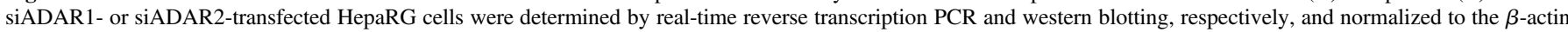

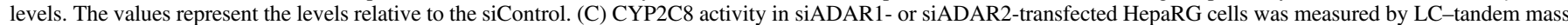
spectrometry. Each column represents the mean \pm S.D. of three independent experiments. $* P<0.05 ; * * P<0.01 ; * * * P<0.001$, compared with siControl. 
A

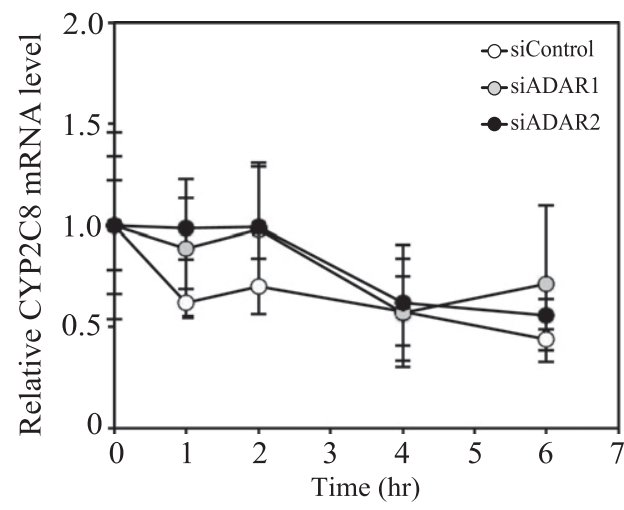

C

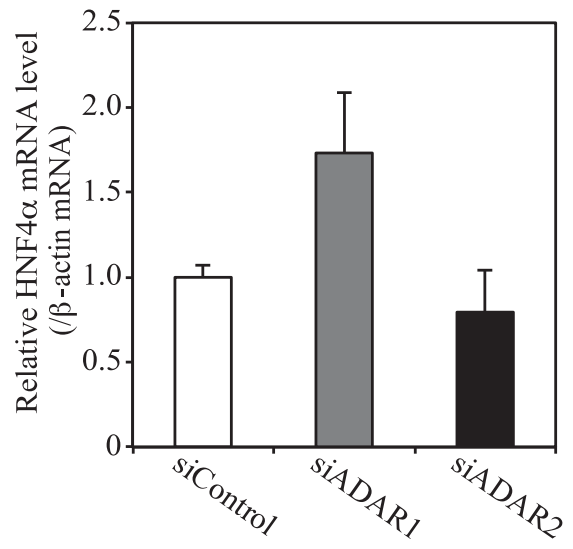

B

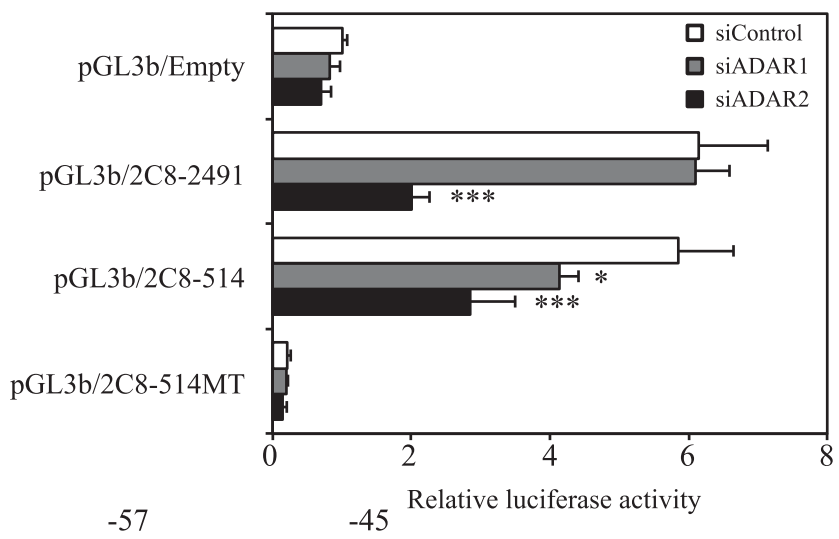

WT 5'-GGGCCAAAGTCCA -3'

MT 5'-GGGCGTTTCACCA -3'

\section{D}

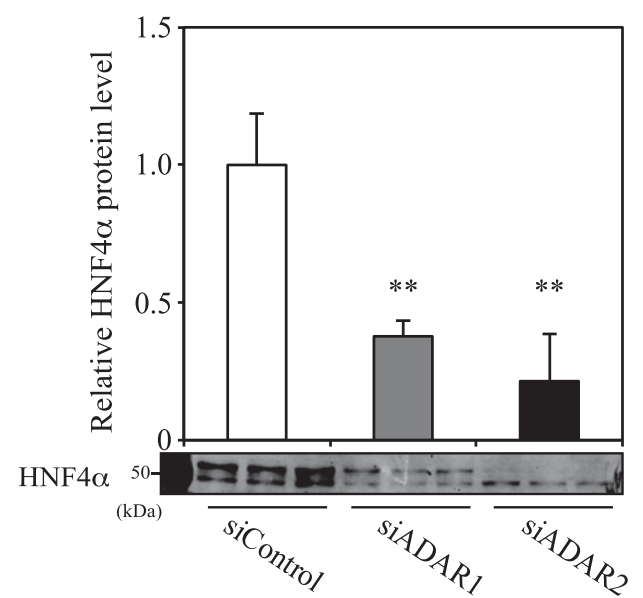

Fig. 5. Effects of ADAR1 or ADAR2 knockdown on the transactivity of CYP2C8. ADARs positively regulate the transactivity of CYP2C8 via HNF4 $\alpha$. (A) siADAR1- or siADAR2-transfected HepaRG cells were treated with $10 \mu \mathrm{g} / \mathrm{ml}$ of $\alpha$-amanitin and CYP2C8 mRNA was determined by real-time reverse transcription PCR (RT-PCR). The values are expressed as relative to the values of the CYP2C8 mRNA levels at 0 hours. Each point represents the mean \pm S.D. of three independent experiments. (B) Huh-7 cells were transfected with $5 \mathrm{nM}$ siADAR1 or siADAR2. After 24 hours, the reporter plasmids were transfected. The values are expressed as the activity relative to that of the pGL3p plasmid/siControl. (C and D) HNF4 $\alpha$ mRNA and protein levels (using nuclear extracts) in siADAR1- or siADAR2-transfected Huh-7 cells were determined by realtime RT-PCR and western blotting, respectively. The HNF4 $\alpha$ protein level was evaluated as the sum of the densities of two bands. The values represent the levels relative to the siControl. Each column represents the mean \pm S.D. of three independent experiments. $* P<0.05 ; * * P<0.01$; $* * * P<0.001$, compared with siControl

and $78 \%$ of siControl, respectively), CYP2A6 (71\% and $72 \%$ of siControl, respectively), CYP2C9 (60\% of siControl), CYP2C19 (76\% and $47 \%$ of siControl, respectively), CYP2D6 (26\% and $40 \%$ of siControl, respectively), CYP2E1 (78\% and $81 \%$ of siControl, respectively), and CYP3A5 (72\% and $40 \%$ of siControl, respectively) mRNA levels were decreased by ADAR1 or ADAR2 knockdown, although the changes in CYP1A2 and CYP2D6 by ADAR1 knockdown and the changes in CYP3A5 by ADAR knockdown did not show significant differences. The CYP3A4 mRNA was decreased $(76 \%$ of siControl) by the knockdown of ADAR2, but unexpectedly, it was increased 3-fold by the knockdown of ADAR1 (Fig. 6G). Next, the effects of ADAR knockdown on the P450 activities were evaluated by using LC-tandem mass spectrometry. We measured phenacetin $O$ deethylation, diclofenac 4'-hydroxylation, and bufuralol 1'-hydroxylation as the typical activities of CYP1A2, CYP2C9, and CYP2D6, respectively. The CYP1A2 activity (24\% of siControl) was not changed by ADAR knockdown (Fig. 6I), whereas the CYP2C9 (44\% siControl) and CYP2D6 (59\% of siControl) activities were decreased by ADAR1 knockdown (Fig. 6, J and K). This phenomenon was consistent with the results of mRNA alteration. Taken together, the results show that the alteration of $\mathrm{HNF} 4 \alpha$ expression affects multiple hepatic P450 mRNA levels. In the case of CYP3A4, there is a possibility that some factors other than $\mathrm{HNF} 4 \alpha$ may also contribute to the change in the expression of ADARs.

\section{Discussion}

A-to-I RNA editing has the potential to change gene expression and function. The effects of RNA editing on the pharmacokinetic-related genes have not yet been clarified. In this study, we investigated the possibility that ADARs regulate human $\mathrm{P} 450$ expression and activity in the liver.

We found that ADAR knockdown resulted in a decrease in CYP2B6 mRNA via enhancing mRNA stability (Fig. 3A). In REDIportal, RNA 
A

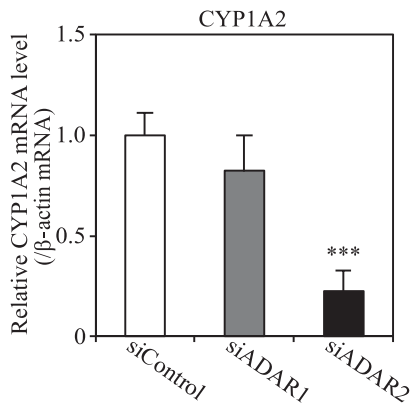

D

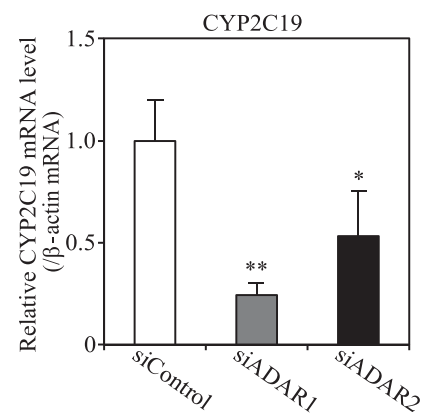

G

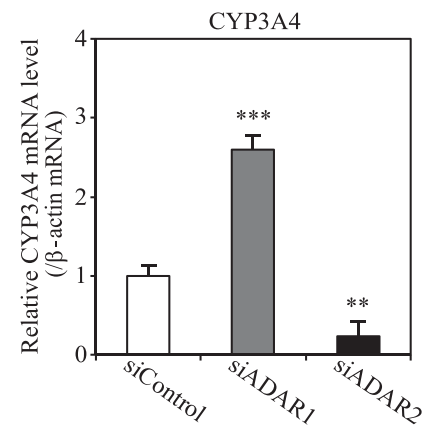

I

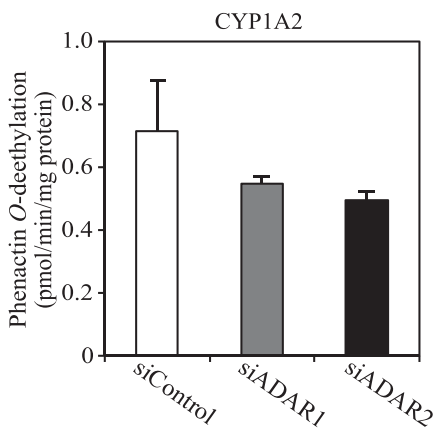

B

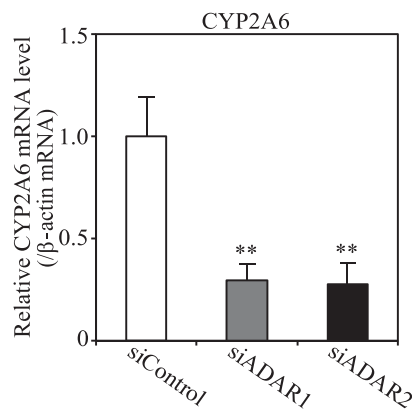

E

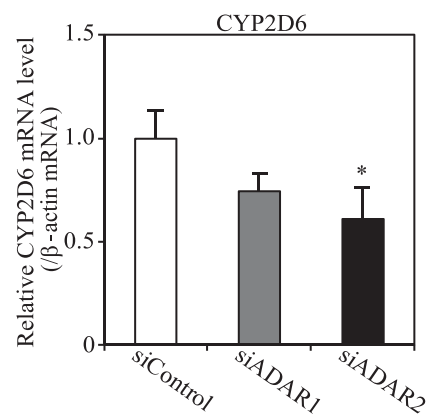

H

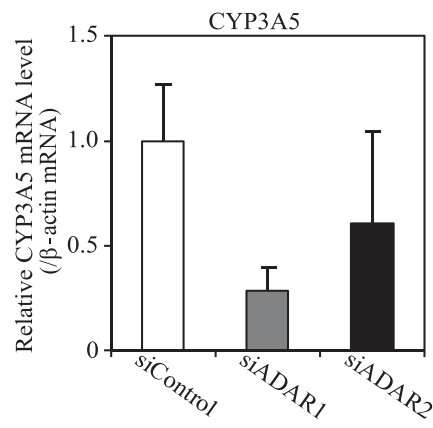

$\mathbf{J}$

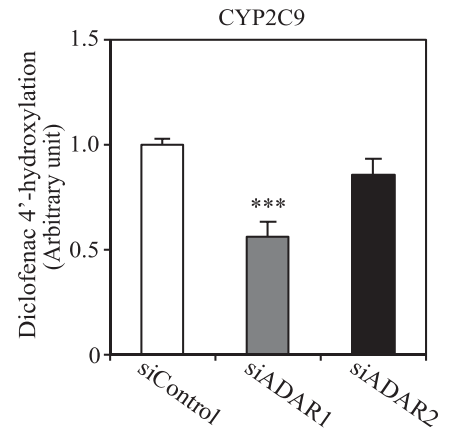

C

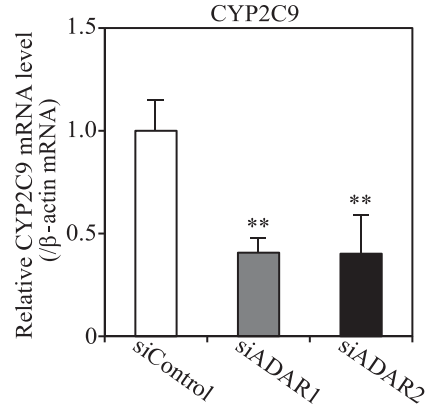

$\mathbf{F}$

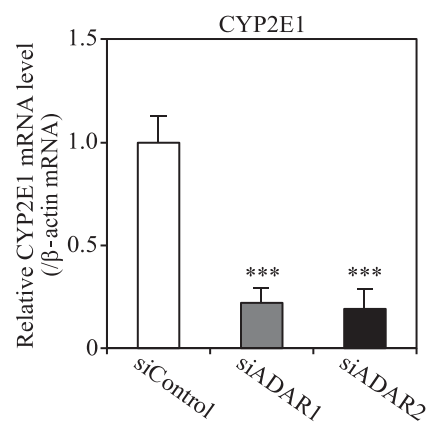

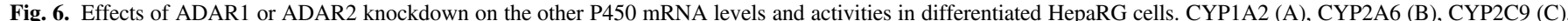

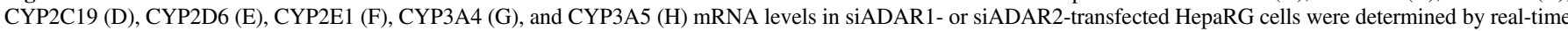

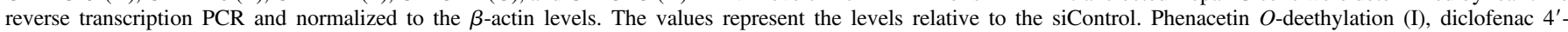

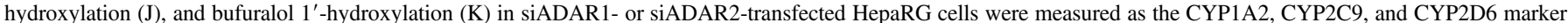
activities, respectively. Each column represents the mean \pm S.D. of three independent experiments. $* P<0.05$; $* * P<0.01 ; * * * P<0.001$, compared with siControl.

editing sites are registered within the two Alu elements in the $3^{\prime}$-UTR of CYP2B6. It has been reported that $\sim 90 \%$ of RNA editing sites are located in Alu elements in the human transcriptome (Picardi et al., 2017).
Because RNA editing in the $3^{\prime}$-UTR may be relevant to the changed stability of mRNA, we examined whether the $3^{\prime}$-UTR of CYP2B6 in HepaRG cells is actually subjected to RNA editing by direct sequencing. 
However, no editing sites were detected in this region (data not shown). Recently, it has been reported that ADARs regulate gene expression in an editing-independent manner. For example, ADARs repressed the expression of methyltransferase such as 7A (METTL7A), a tumor suppressor gene, by promoting the processing of miR-27a that targets the 3'-UTR of METTL7A. This phenomenon was also observed in an ADAR mutant lacking enzymatic activity, indicating that ADARs regulate METTL7A in an editing-independent manner (Qi et al., 2017). In another paper, it was reported that ADAR2 enhances the stability of nuclear-retained, Cat2-transcribed nuclear RNA by limiting the access of the RNA binding protein $\mathrm{HuR}$ and poly(A) specific ribonuclease deadenylase (Anantharaman et al., 2017). It is worth investigating the possibility that CYP2B6 is regulated in an editingindependent manner. In addition, there is a possibility that ADARs regulate CYP2B6 at the transcriptional level as well as the posttranscriptional level because the alteration of CYP2B6 mRNA stability by knockdown of ADARs was somewhat small (Fig. 3A). It has been reported that CYP2B6 is transactivated by HNF4 $\alpha$ (Kamiyama et al., 2007), whose expression was decreased by knockdown of ADARs (Fig. 5D). Therefore, the regulation of $\mathrm{HNF} 4 \alpha$ by ADAR1 possibly contributes CYP2B6 expression.

We found that CYP2C8 expression and activity were decreased by ADAR knockdown (Fig. 4), and this alteration occurred in a transcriptional manner (Fig. 5A). It has been reported that CYP2C8 upstream has the response elements of peroxisome proliferator-activated receptor $\alpha$, glucocorticoid receptor, and HNF4 $\alpha$ (Ferguson et al., 2005; Makia and Goldstein, 2016). The luciferase activity of the pGL3b/2C8-514 plasmid was decreased by ADAR1 or ADAR2 knockdown (Fig. 5B). When mutations were introduced in the response element of $\mathrm{HNF} 4 \alpha$, the luciferase activity was not changed by the knockdown of ADAR1 or ADAR2 (Fig. 5B). Additionally, the HNF4 $\alpha$ protein level was also decreased by ADAR1 or ADAR2 knockdown (Fig. 5D). Thus, it was suggested that ADAR1 or ADAR2 positively regulates the expression of CYP2C8 through the upregulation of HNF4 $\alpha$. Since the HNF4 $\alpha$ mRNA level was not changed by ADAR1 or ADAR2 knockdown (Fig. 5C), it was believed that ADARs may positively regulate HNF $4 \alpha$ expression in a post-transcriptional manner. According to REDIportal, HNF4 $\alpha$ mRNA does not have any RNA editing sites, indicating that ADARs indirectly regulate $\mathrm{HNF} 4 \alpha$ expression. Some miRNAs have been reported to regulate $\mathrm{HNF} 4 \alpha$ expression (Takagi et al., 2010; Ramamoorthy et al., 2012; Wang and Burke et al., 2013), and A-to-I changes in an miRNA transcript can alter its processing to cause changes in mature miRNA expression (Ekdahl et al., 2012; Vesely et al., 2012). Therefore, it is possible that ADARs regulate HNF $4 \alpha$ by changing the miRNA processing.

CYP2C8 plays a role in the metabolism of clinically used drugs, such as paclitaxel (Rahman et al., 1994), amodiaquine (Walsky and Obach, 2004), and amiodarone (Ohyama et al., 2000). Since the CYP2C8 activity was decreased by ADAR1 knockdown, the alteration of ADAR1 expression may influence the metabolism of these drugs; this results in alterations to the drug efficacy or causes adverse effects. There are large interindividual differences in the CYP2C8 protein level and activity (Naraharisetti et al., 2010), and the variability is partially attributed to the genetic polymorphism of $C Y P 2 C 8$. Among the $14 C Y P 2 C 8$ alleles, $C Y P 2 C 8 * 2, C Y P 2 C 8 * 3$, and $C Y P 2 C 8 * 4$ are common alleles with amino acid changes. These alleles have been reported to contribute to alterations in CYP2C8 activity. For example, liver samples from heterozygotes of $C Y P 2 C 8 * 3$ showed significantly lower paclitaxel $6 \alpha$ hydroxylase activity compared with wild-type samples (Bahadur et al., 2002). In contrast, another research group reported no difference in the activities between two groups (Taniguchi et al., 2005). There is a large interindividual difference in the CYP2C8 metabolic activities among the same genotype; therefore, it is possible that the interindividual variation could be due to factors other than genetic factors. The present study revealed that CYP2C8 expression was modulated by ADARs (Fig. 4). The ADAR-dependent regulation of $\mathrm{CYP} 2 \mathrm{C} 8$ also contributes to the interindividual differences in CYP2C8.

Since the CYP2A6, 2B6, 2C9, 2C19, 2D6, 2E1, and 3A4 expression levels are also regulated by HNF $4 \alpha$ (Kamiyama et al., 2007; Chen et al., 2018), these P450 mRNA levels were evaluated. We found that the CYP2A6, 2C9, 2C19, 2D6, and 3A4 mRNA levels were decreased by ADAR1 or ADAR2 knockdown (Fig. 6, B-F), and these alterations were consistent with HNF4 $\alpha$ expression (Fig. 5E). Thus, HNF4 $\alpha$ could be involved in the decreases in these P450 mRNA levels by ADAR1 or ADAR2 knockdown. Unlike other P450 isoforms, the CYP3A4 mRNA levels were significantly increased by the knockdown of ADAR1 (Fig. $6 \mathrm{G})$. The change was not consistent with that of HNF4 $\alpha$ expression. In a recent paper, it was shown that the contribution of $\mathrm{HNF} 4 \alpha$ in the regulation of CYP3A4 is smaller than that of the other P450 isoforms (Chen et al., 2018).

In conclusion, the present study has demonstrated that the RNA editing enzymes, ADARs, are novel factors regulating P450. Our previous study revealed a large interindividual variation (220-fold) in ADAR1 protein expression in human liver samples (Nakano et al., 2016). Therefore, the variation in ADAR1 expression could be one of the factors causing the interindividual variation in $\mathrm{P} 450$ expression.

\section{Authorship Contributions}

Participated in research design: Nozaki, Nakano, Fukami, Nakajima.

Conducted experiments: Nozaki.

Contributed new reagents or analytic tools: Nozaki, Iwakami.

Performed data analysis: Nozaki, Nakano, Nakajima.

Wrote or contributed to the writing of the manuscript: Nozaki, Nakano, Nakajima.

\section{References}

Anantharaman A, Tripathi V, Khan A, Yoon JH, Singh DK, Gholamalamdari O, Guang S, Ohlson J, Wahlstedt H, Öhman M, et al. (2017) ADAR2 regulates RNA stability by modifying access of decay-promoting RNA-binding proteins. Nucleic Acids Res 45:4189-4201.

Anthérieu S, Chesné C, Li R, Camus S, Lahoz A, Picazo L, Turpeinen M, Tolonen A, Uusitalo J, Guguen-Guillouzo C, et al. (2010) Stable expression, activity, and inducibility of cytochromes P450 in differentiated HepaRG cells. Drug Metab Dispos 38:516-525.

Bahadur N, Leathart JBS, Mutch E, Steimel-Crespi D, Dunn SA, Gilissen R, Houdt JV, Hendrickx J, Mannens G, Bohets H, et al. (2002) CYP2C8 polymorphisms in Caucasians and their relationship with paclitaxel $6 \alpha$-hydroxylase activity in human liver microsomes. Biochem Pharmacol 64:1579-1589.

Bass BL, Nishikura K, Keller W, Seeburg PH, Emeson RB, O'Connell MA, Samuel CE, and Herbert A (1997) A standardized nomenclature for adenosine deaminases that act on RNA. RNA 3:947-949.

Chen CX, Cho DS, Wang Q, Lai F, Carter KC, and Nishikura K (2000) A third member of the RNA-specific adenosine deaminase gene family, ADAR3, contains both single- and doublestranded RNA binding domains. RNA 6:755-767.

Chen L, Bao Y, Piekos SC, Zhu K, Zhang L, and Zhong XB (2018) A transcriptional regulatory network containing nuclear receptors and long noncoding RNAs controls basal and drug-induced expression of cytochrome P450s in HepaRG cells. Mol Pharmacol 94:749-759.

Desterro JMP, Keegan LP, Lafarga M, Berciano MT, O'Connell M, and Carmo-Fonseca M (2003) Dynamic association of RNA-editing enzymes with the nucleolus. J Cell Sci 116:1805-1818.

Dignam JD, Lebovitz RM, and Roeder RG (1983) Accurate transcription initiation by RNA polymerase II in a soluble extract from isolated mammalian nuclei. Nucleic Acids Res 11: $1475-1489$.

Ekdahl Y, Farahani HS, Behm M, Lagergren J, and Öhman M (2012) A-to-I editing of microRNAs in the mammalian brain increases during development. Genome Res 22:1477-1487.

Farajollahi S and Maas S (2010) Molecular diversity through RNA editing: a balancing act. Trends Genet 26:221-230.

Feldmeyer D, Kask K, Brusa R, Kornau HC, Kolhekar R, Rozov A, Burnashev N, Jensen V, Hvalby O, Sprengel R, et al. (1999) Neurological dysfunctions in mice expressing different levels of the Q/R site-unedited AMPAR subunit GluR-B. Nat Neurosci 2:57-64.

Ferguson SS, Chen Y, LeCluyse EL, Negishi M, and Goldstein JA (2005) Human CYP2C8 is transcriptionally regulated by the nuclear receptors constitutive androstane receptor, pregnane $\mathrm{X}$ receptor, glucocorticoid receptor, and hepatic nuclear factor $4 \alpha$. Mol Pharmacol 68:747-757.

Guengerich FP (2008) Cytochrome P450 and chemical toxicology. Chem Res Toxicol 21:70-83.

Higuchi M, Maas S, Single FN, Hartner J, Rozov A, Burnashev N, Feldmeyer D, Sprengel R, and Seeburg PH (2000) Point mutation in an AMPA receptor gene rescues lethality in mice deficient in the RNA-editing enzyme ADAR2. Nature 406:78-81.

Iwanari M, Nakajima M, Kizu R, Hayakawa K, and Yokoi T (2002) Induction of CYP1A1, CYP1A2, and CYP1B1 mRNAs by nitropolycyclic aromatic hydrocarbons in various human 
tissue-derived cells: chemical-, cytochrome P450 isoform-, and cell-specific differences. Arch Toxicol 76:287-298.

Jin Y, Yu D, Tolleson WH, Knox B, Wang Y, Chen S, Ren Z, Deng H, Guo Y, and Ning B (2016) MicroRNA hsa-miR-25-3p suppresses the expression and drug induction of CYP2B6 in human hepatocytes. Biochem Pharmacol 113:88-96.

Kamiyama Y, Matsubara T, Yoshinari K, Nagata K, Kamimura H, and Yamazoe Y (2007) Role of human hepatocyte nuclear factor $4 \alpha$ in the expression of drug-metabolizing enzymes and transporters in human hepatocytes assessed by use of small interfering RNA. Drug Metab Pharmacokinet 22:287-298.

Katoh M, Matsui T, Nakajima M, Tateno C, Kataoka M, Soeno Y, Horie T, Iwasaki K, Yoshizato $\mathrm{K}$, and Yokoi T (2004) Expression of human cytochromes P450 in chimeric mice with humanized liver. Drug Metab Dispos 32:1402-1410.

Kim U, Wang Y, Sanford T, Zeng Y, and Nishikura K (1994) Molecular cloning of cDNA for double-stranded RNA adenosine deaminase, a candidate enzyme for nuclear RNA editing. Proc Natl Acad Sci USA 91:11457-11461.

Makia NL and Goldstein JA (2016) CYP2C8 is a novel target of peroxisome proliferator-activated receptor $\alpha$ in human liver. Mol Pharmacol 89:154-164.

Melcher T, Maas S, Herb A, Sprengel R, Higuchi M, and Seeburg PH (1996) RED2, a brainspecific member of the RNA-specific adenosine deaminase family. $J$ Biol Chem 271 31795-31798.

Miyamura Y, Suzuki T, Kono M, Inagaki K, Ito S, Suzuki N, and Tomita Y (2003) Mutations of the RNA-specific adenosine deaminase gene $(D S R A D)$ are involved in dyschromatosis symmetrica hereditaria. Am J Hum Genet 73:693-699.

Mohri T, Nakajima M, Fukami T, Takamiya M, Aoki Y, and Yokoi T (2010) Human CYP2E1 is regulated by miR-378. Biochem Pharmacol 79:1045-1052.

Nakajima M, Itoh M, Sakai H, Fukami T, Katoh M, Yamazaki H, Kadlubar FF, Imaoka S, Funae Y, and Yokoi T (2006) CYP2A13 expressed in human bladder metabolically activates 4-aminobiphenyl. Int J Cancer 119:2520-2526.

Nakano M, Fukami T, Gotoh S, Takamiya M, Aoki Y, and Nakajima M (2016) RNA editing modulates human hepatic aryl hydrocarbon receptor expression by creating microRNA recognition sequence. J Biol Chem 291:894-903.

Nakano M and Nakajima M (2018) Significance of A-to-I RNA editing of transcripts modulating pharmacokinetics and pharmacodynamics. Pharmacol Ther 181:13-21.

Naraharisetti SB, Lin YS, Rieder MJ, Marciante KD, Psaty BM, Thummel KE, and Totah RA (2010) Human liver expression of CYP2C8: gender, age, and genotype effects. Drug Metab Dispos 38:889-893.

Nishikura K (2010) Functions and regulation of RNA editing by ADAR deaminases. Anпи Rev Biochem 79:321-349.

Oda Y, Nakajima M, Mohri T, Takamiya M, Aoki Y, Fukami T, and Yokoi T (2012) Aryl hydrocarbon receptor nuclear translocator in human liver is regulated by miR-24. Toxicol Appl Pharmacol 260:222-231.

Ohyama K, Nakajima M, Nakamura S, Shimada N, Yamazaki H, and Yokoi T (2000) A significan role of human cytochrome P450 2C8 in amiodarone $N$-deethylation: an approach to predict the contribution with relative activity factor. Drug Metab Dispos 28:1303-1310.
Patterson JB and Samuel CE (1995) Expression and regulation by interferon of a double-strandedRNA-specific adenosine deaminase from human cells: evidence for two forms of the deaminase. Mol Cell Biol 15:5376-5388.

Picardi E, D'Erchia AM, Lo Giudice C, and Pesole G (2017) REDIportal: a comprehensive database of A-to-I RNA editing events in humans. Nucleic Acids Res 45:D750-D757.

Qi L, Song Y, Chan THM, Yang H, Lin CH, Tay DJT, Hong H, Tang SJ, Tan KT, Huang XX, et al (2017) An RNA editing/dsRNA binding-independent gene regulatory mechanism of ADARs and its clinical implication in cancer. Nucleic Acids Res 45:10436-10451.

Rahman A, Korzekwa KR, Grogan J, Gonzalez FJ, and Harris JW (1994) Selective biotransformation of taxol to $6 \alpha$-hydroxytaxol by human cytochrome P450 2C8. Cancer Res 54 $5543-5546$.

Ramamoorthy A, Li L, Gaedigk A, Bradford LD, Benson EA, Flockhart DA, and Skaar TC (2012) In silico and in vitro identification of microRNAs that regulate hepatic nuclear factor $4 \alpha$ expression. Drug Metab Dispos 40:726-733.

Slotkin W and Nishikura K (2013) Adenosine-to-inosine RNA editing and human disease. Genome Med 5:105.

Takagi S, Nakajima M, Kida K, Yamaura Y, Fukami T, and Yokoi T (2010) MicroRNAs regulate human hepatocyte nuclear factor $4 \alpha$, modulating the expression of metabolic enzymes and cell cycle. J Biol Chem 285:4415-4422.

Taniguchi R, Kumai T, Matsumoto N, Watanabe M, Kamio K, Suzuki S, and Kobayashi S (2005) Utilization of human liver microsomes to explain individual differences in paclitaxel metabolism by CYP2C8 and CYP3A4. J Pharmacol Sci 97:83-90.

Vesely C, Tauber S, Sedlazeck FJ, von Haeseler A, and Jantsch MF (2012) Adenosine deaminases that act on RNA induce reproducible changes in abundance and sequence of embryonic miRNAs. Genome Res 22:1468-1476.

Wagner RW, Smith JE, Cooperman BS, and Nishikura K (1989) A double-stranded RNA unwinding activity introduces structural alterations by means of adenosine to inosine conversions in mammalian cells and Xenopus eggs. Proc Natl Acad Sci USA 86:2647-2651.

Walsky RL and Obach RS (2004) Validated assays for human cytochrome P450 activities. Drug Metab Dispos 32:647-660.

Wang Q, Khillan J, Gadue P, and Nishikura K (2000) Requirement of the RNA editing deaminase ADAR1 gene for embryonic erythropoiesis. Science 290:1765-1768.

Wang Z and Burke PA (2013) The role of microRNAs in hepatocyte nuclear factor- $4 \alpha$ expression and transactivation. Biochim Biophys Acta 1829:436-442.

Zanger UM and Schwab M (2013) Cytochrome P450 enzymes in drug metabolism: regulation of gene expression, enzyme activities, and impact of genetic variation. Pharmacol Ther 138:103-141.

Address correspondence to: Dr. Miki Nakajima, Drug Metabolism and Toxicology, Faculty of Pharmaceutical Sciences/WPI Nano Life Science Institute, Kanazawa University, Kakuma-machi, Kanazawa 920-1192, Japan. E-mail: nmiki@p.kanazawa-u.ac.jp 